\title{
Analysis of the Working Principle of 12 Pulse Inverter Bridge
}

\author{
Zhou Guangyang ${ }^{1}$ \\ ${ }^{1}$ School of Electrical and Electric Engineering, North China Electric Power University, Beijing, 102206, \\ China
}

Keywords: Inverter, HVDC, Voltage, Current, Formula

\begin{abstract}
This paper mainly introduces the working principle of double 12 pulse inverter. By analyzing the operation process of the inverter, obtaining the expression of voltage and current in both DC side and AC side respectively, and getting the relevant calculation formula of angle. Connecting two a 6 pulse converter in the DC side, we get a 12 pulse converter. Its AC side of the converter is connected in parallel through the converter transformer.Valve side of converter transformer, one is connected into a star, and the other is connected to a triangle, thus the phase difference of the exchange side is 30 degrees. The normal operation of the inverter bridge is 4-5 working states, besides there are non-normal operation modes such as 5 working states, 5-6 working states and 6 working states, etc.
\end{abstract}

\section{Normal Operation Mode- 4-5 Working State}

Taking two groups of double winding converter transformer as an example, we will analysis the working principle of double 12 pulse inverter. Assuming that the Yy connection of the converter transformer is ahead of the Yd connection of the converter transformer, so, for the same valve arm, the bridge 1 is 30 degrees ahead of bridge 2. But for each of the 6 valve arms within a single bridge, they still turn on spaced at 60 degrees. So, the conducting sequence of the 12 valve arm is: $\mathrm{VT}_{11}$, $\mathrm{VT}_{12}, \mathrm{VT}_{21}, \mathrm{VT}_{22}, \mathrm{VT}_{31}, \mathrm{VT}_{32}, \mathrm{VT}_{41}, \mathrm{VT}_{42}, \mathrm{VT}_{51}, \mathrm{VT}_{52}, \mathrm{VT}_{61}, \mathrm{VT}_{62}, \mathrm{VT}_{11} \ldots$ (repeating the cycle), each valve is guided by an interval of 30 degrees. As shown in Figure 1, the first number of the valve armrepresents the order in its own bridge, while the second number represents the number of bridges. 


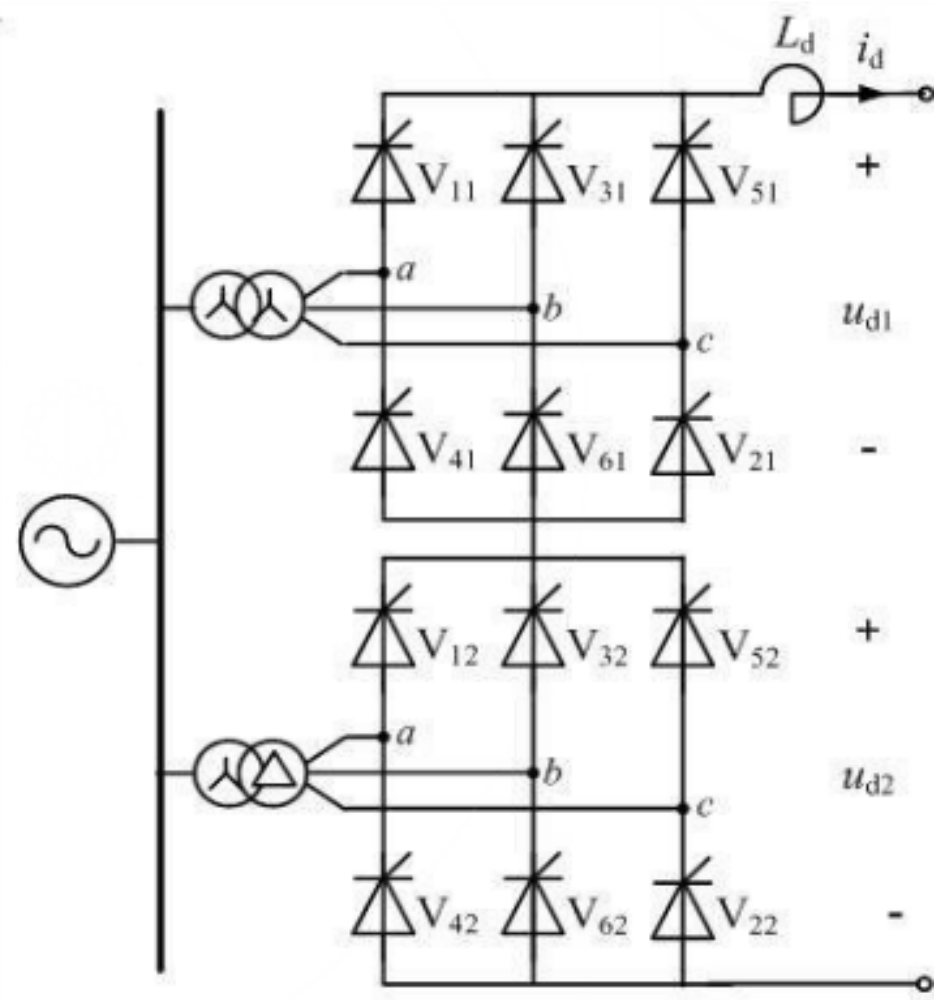

Figure 1 Double 12 pulse inverter principle diagram

The 4-5 working state means a mode in a complete working cycle, 4 valve arms and 5 valve arms alternately conducting operation. The 12 valve arms are in the order of: $\mathrm{VT}_{11}, \mathrm{VT}_{12}, \mathrm{VT}_{21}$, $\mathrm{VT}_{22} \rightarrow \mathrm{VT}_{11}, \mathrm{VT}_{12}, \mathrm{VT}_{21}, \mathrm{VT}_{22}, \mathrm{VT}_{31} \rightarrow \mathrm{VT}_{31}, \mathrm{VT}_{12}, \mathrm{VT}_{21}, \mathrm{VT}_{22} \rightarrow \mathrm{VT}_{31}, \mathrm{VT}_{12}, \mathrm{VT}_{21}, \mathrm{VT}_{22}, \mathrm{VT}_{31} \rightarrow$ $\mathrm{VT}_{21}, \quad \mathrm{VT}_{22}, \quad \mathrm{VT}_{31}, \quad \mathrm{VT}_{32} \rightarrow \mathrm{VT}_{21} \mathrm{VT}_{22}, \mathrm{VT}_{31}, \quad \mathrm{VT}_{32}, \quad \mathrm{VT}_{41} \rightarrow \mathrm{VT}_{22}, \quad \mathrm{VT}_{31}, \quad \mathrm{VT}_{32}, \quad \mathrm{VT}_{41}$ $\rightarrow \mathrm{VT}_{22}, \mathrm{VT}_{31}, \mathrm{VT}_{32}, \mathrm{VT}_{41}, \mathrm{VT}_{42} \rightarrow \mathrm{VT}_{31}, \mathrm{VT}_{32}, \mathrm{VT}_{41}, \mathrm{VT}_{42} \rightarrow \mathrm{VT}_{31}, \mathrm{VT}_{32}, \mathrm{VT}_{41}, \mathrm{VT}_{42}, \mathrm{VT}_{51} \rightarrow \mathrm{VT}_{32}$, $\mathrm{VT}_{41}, \mathrm{VT}_{42}, \mathrm{VT}_{51} \rightarrow \mathrm{VT}_{32}, \mathrm{VT}_{41}, \mathrm{VT}_{42}, \mathrm{VT}_{51}, \mathrm{VT}_{52} \rightarrow \mathrm{VT}_{41}, \mathrm{VT}_{42}, \mathrm{VT}_{51}, \mathrm{VT}_{52} \rightarrow \mathrm{VT}_{41}, \mathrm{VT}_{42}, \mathrm{VT}_{51}$, $\mathrm{VT}_{52}, \mathrm{VT}_{61} \rightarrow \mathrm{VT}_{42}, \mathrm{VT}_{51}, \mathrm{VT}_{52}, \mathrm{VT}_{61} \rightarrow \mathrm{VT}_{42}, \mathrm{VT}_{51}, \mathrm{VT}_{52}, \mathrm{VT}_{61}, \mathrm{VT}_{62} \rightarrow \mathrm{VT}_{51}, \mathrm{VT}_{52}, \mathrm{VT}_{61}, \mathrm{VT}_{62}$ $\rightarrow \mathrm{VT}_{51}, \mathrm{VT}_{52}, \mathrm{VT}_{61}, \mathrm{VT}_{62}, \mathrm{VT}_{11} \rightarrow \mathrm{VT}_{52}, \mathrm{VT}_{61}, \mathrm{VT}_{62}, \mathrm{VT}_{11} \rightarrow \mathrm{VT}_{52}, \mathrm{VT}_{61}, \mathrm{VT}_{62}, \mathrm{VT}_{11}, \mathrm{VT}_{12} \rightarrow \mathrm{VT}_{61}$, $\mathrm{VT}_{62}, \mathrm{VT}_{11}, \mathrm{VT}_{12} \rightarrow \mathrm{VT}_{61}, \mathrm{VT}_{62}, \mathrm{VT}_{11}, \mathrm{VT}_{12}, \mathrm{VT}_{21} \rightarrow \mathrm{VT}_{62}, \mathrm{VT}_{11}, \mathrm{VT}_{12}, \mathrm{VT}_{21} \rightarrow \mathrm{VT}_{62}, \mathrm{VT}_{11}, \mathrm{VT}_{12}$, $\mathrm{VT}_{21}, \mathrm{VT}_{22} \mathrm{VT}_{11}, \mathrm{VT}_{12}, \mathrm{VT}_{21}, \mathrm{VT}_{22} \rightarrow$ (loop).

The condition of 12 pulse inverter is: 1). External DC power supply, and the polarity of the power supply is consistent with the conduction direction of the thyristor. 2). The average value of the rectifier voltage of the external communication systemshould be less than DC power supply voltage. 3). The trigger angle of thyristoro should be continuous adjustable in the range of 90-180 degrees. The prerequisite for double 12 pulse inverter working in the condition of $4-5 i s 90^{\circ}-\frac{\mu}{2}<\alpha \leq 180^{\circ}$, at the same time, the commutation $0^{\circ} \leq \mu<30^{\circ}$. If $90^{\circ}-\frac{\mu}{2}<\alpha \leq 180^{\circ}, \mu \geq 30^{\circ}$, inverter will work in 5-6 condition, which is an abnormal operating condition.

\section{Related Calculation Formula}

The average value of the inverter bridge rectifier voltage

$$
\begin{gathered}
\mathrm{U}_{d}=U_{d 1}+U_{d 2}=2 U_{d 0} \frac{\cos \beta+\cos (\beta+\mu)}{2} \\
U_{d}=2 U_{d 0} \cos \left(\beta-\frac{\mu}{2}\right) \cdot \cos \frac{\mu}{2}
\end{gathered}
$$




$$
U_{d}=2\left(U_{d 0} \cos \gamma-\frac{3 \omega L_{r}}{\pi} i_{d}\right)
$$

In the above equation, $\mathrm{U}_{\mathrm{d} 0}$ is single bridge ideal no-load $\mathrm{DC}$ voltage, its calculation is:

$$
U_{d 0}=\frac{3 \sqrt{2}}{\pi} E \approx 1.35 E
$$

E represents effective value of AC line voltage.

Harmonics in voltage

The rectifier voltage of each bridge forming the double bridge contains 6k-th harmonic, while the $6 *(2 \mathrm{k}-1)$-th harmonic generated by bridge. 1 and bridge. 2 are equal to the harmonic current but the polarity is opposite, so they all cancel each other out. Therefor, the rectifier voltage of a 12 pulse inverter only contains the following number of harmonics:

$$
h=12 k
$$

In the above equation, $k=1,2,3, \ldots$

That is to say, double 12 pulse inverter only consists with $12^{\text {nd }}, 24^{\text {th }}, 36^{\text {th }} \ldots$ harmonic.

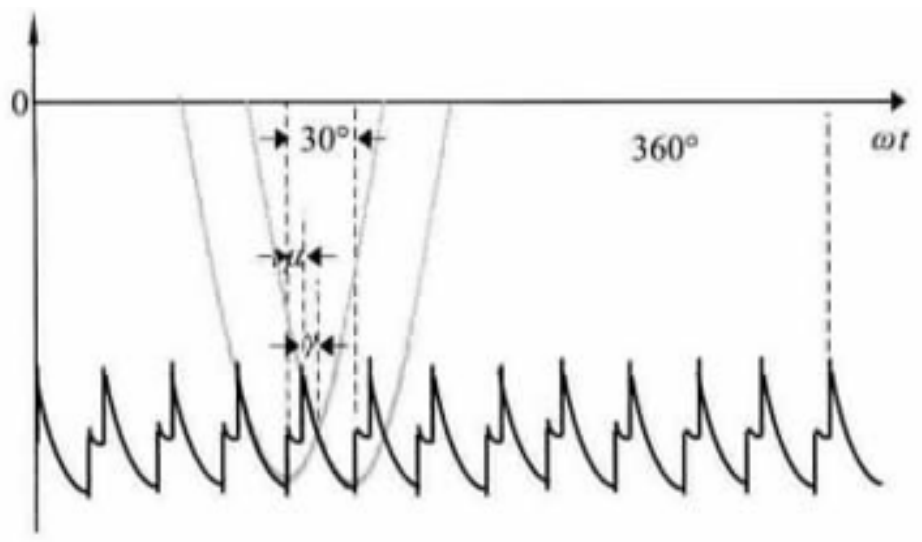

Figure 2 Double 12 pulse inverter voltage waveform

Current calculation formula

The Fourier series expansion of the A phase current inYy connected converter transformer's valve side, network side and Yd connected converter transformer valve side is:

$$
\mathrm{i}_{A 1}=i_{a 1}=\frac{2 \sqrt{3}}{\pi} i_{d}\left[\cos \omega t-\frac{1}{5} \cos 5 \omega t+\frac{1}{7} \cos 7 \omega t-\frac{1}{11} \cos 11 \omega t \cdots\right]
$$

The Fourier series expansion of the A phase current in network side of converter transformer with Yd connection is:

$$
i_{A 2}=\frac{2 \sqrt{3}}{\pi} i_{d}\left[\cos \omega t+\frac{1}{5} \cos 5 \omega t-\frac{1}{7} \cos 7 \omega t-\frac{1}{11} \cos 11 \omega t \cdots\right]
$$

The Fourier series expansion of the A phase current in communication system is:

$$
i_{A}=\frac{4 \sqrt{3}}{\pi} i_{d}\left[\cos \omega t-\frac{1}{11} \cos 11 \omega t+\frac{1}{13} \cos 13 \omega t-\frac{1}{23} \cos 23 \omega t+\frac{1}{25} \cos 25 \omega t-\cdots\right]
$$

As a result, the following conclusions can be drawn:

The network side of converter transformer contains $h=(6 k \pm 1)^{\text {th }}$ harmonic. 
The current of the AC side power system contains $h=(12 k \pm 1)^{\text {th }}$ harmonics.

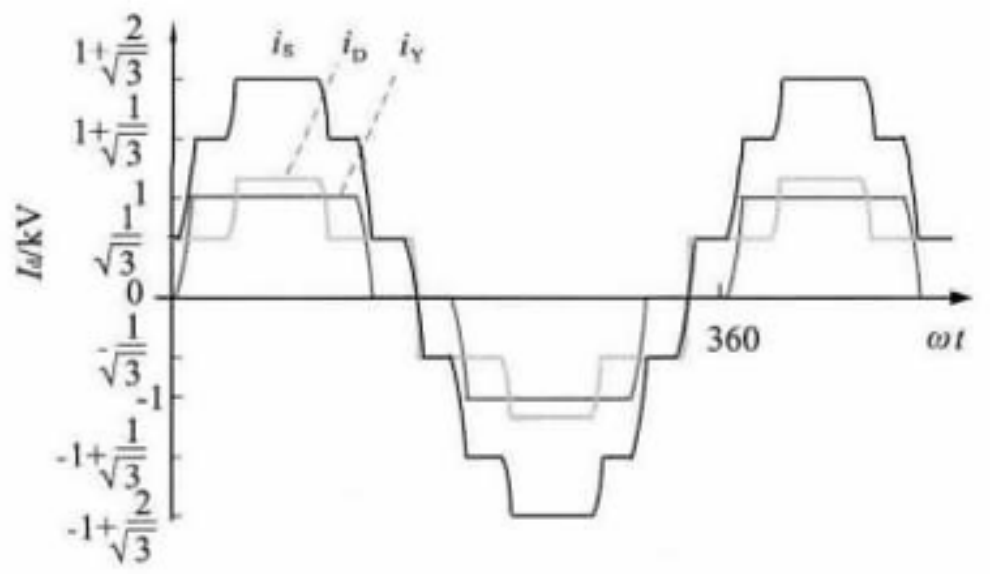

Figure 3 Double 12 pulse inverter current waveform

The external characteristic of inverter bridge equation

The outer characteristic equation of $\gamma$ :

$$
U_{\mathrm{d}}=U_{d 1}+U_{d 2}=U_{d 0} \cos \gamma-R I_{d}
$$

The outer characteristic equation of $\beta$ :

$$
U_{d}=U_{d 1}+U_{d 2}=U_{d 0} \cos \beta+R I_{d}
$$

The formula in inverter bridge of $\mu$ :

$$
\begin{gathered}
\cos (\gamma+\mu)=\cos \gamma-\frac{I_{d r}}{I_{S C i 2}} \\
I_{S C r 2}=\frac{E}{\sqrt{2} \omega L_{c r}}
\end{gathered}
$$

or

$$
\cos (\beta-\mu)=\cos \beta+\frac{I_{d}}{I_{S C i 2}}
$$

The formula in inverter bridge of $\varphi$ :

$$
\varphi \approx \gamma+\frac{\mu}{2}
$$

or

$$
\varphi \approx 180^{\circ}-\left(\beta-\frac{\mu}{2}\right)
$$

\section{References}

[1] Yang Hao. Application technology of DC power grid in China [J]. Shaanxi electric power, 2014,01:1-6.

[2] Ma Weimin, Wu Fangjie, Yang Yiming, Zhang Tao. Analysis of the current status and application prospect of flexible HVDC technology [J]. high voltage technology, 2014,08:2429-2439.

[3] Zhang Wenliang, Tang Yong, Zeng Nanchao. The multi terminal transmission technology and 
application prospect of high voltage DC [J]. power system technology, 2010,09:1-6.

[4], two Yong, Sun Shukun, wangchu, leaf garden. High voltage direct current (HVDC) transmission system use the she multi pulse inverter [J]. High voltage technology, 2006,05:77-79+83.

[5] Lu Jin Du, Wang Zhijie, Wang Haiqun, Huang Qiyuan, Wang Haoqing, Du Bin. Research on the technology and application of flexible HVDC [J]. Journal of electric power, 2015,04:293-300.

[6] is Zhang Wei, Ming Hui Wang, Deng Wenbin on Jun Zhu. 500kV converter station for flow valve structure analysis. High voltage apparatus, 2012,07:22-26+30.

[7] Wen Jun, Yin Weiyang, Wen Jialiang, min Xiao Han. High voltage direct current (HVDC) transmission system for streaming technology: a review [J]. China Southern Power Grid technology, 2015,02:16-24. 